\title{
Non-linear settlements of shallow foundation
}

\author{
Viktor Yarkin ${ }^{1, *}$, Hanna Kukhar $^{1}$, and Nataliia Lobacheva ${ }^{2}$ \\ ${ }^{1}$ Donbas National Academy of Civil Engineering and Architecture, 2, Derzhavina Str., \\ Makeyevka, DPR, 286123 \\ ${ }^{2}$ Moscow State University of Civil Engineering, Yaroslavskoe shosse, 26, Moscow, 129337, Russia
}

\begin{abstract}
This article proposes a method for determining the nonlinear settlements of the foundation, based on the method of layer-by-layer summation, which allows to determine the settlement of the foundation until the base bearing capacity is exhausted, taking into account the heterogeneity of the geological structure of the soil massif and the presence of weak underlying layers of soil. The recommended equation for determining the coefficient $k_{s}$ is given, which allows you to approximate almost any curve of the dependence of settlement on pressure, taking into account the properties of the soil base, size, shape and depth of the foundation. It carried out a comparative analysis of the proposed method with the results obtained experimentally and other calculating methods.
\end{abstract}

\section{Introduction}

Non-linear models of the soil foundation were considered in the works of Zaretskii Y.K. [1], Bugrov A.K. [2], Shapiro D.M. [3], Malyshev M.V. [4], Gol'dshtein M.N., Kushner S.G. [5,6], Klepikov S.N. [7], Pilyagin A.V. [8], Fadeev A.B. [9], Kirichek Yu.A., Tregub A.V. [10], I.L. Kristić, V. Szavits-Nossan, P. Miščević [11] and others.

Recently, numerical methods for determining nonlinear settlements by the finite element method (FEM) using various elastoplastic models of the soil base have become increasingly popular $[2,8,9,12]$. Nevertheless, analytical methods of calculation and at the moment have not lost their relevance, since they are most fully developed in regulatory documents and are well confirmed by experimental data.

In practice, as before, simplified solutions are widespread, which is sufficiently justified by the inappropriateness of a rigorous mathematical solution with insufficient reliability of determining the initial data on the mechanical behavior of soils [13]. Many of them are based on the method of layer-by-layer summation, which continues to be improved through the use of the deformation characteristics of the soil, determined taking into account its stress state [14], it is possible to significantly improve the accuracy of the determination of the settlement.

Below are some simplified solutions based on the description of the relationship between pressure and deformation in the phase shear of soil (at pressures exceeding the design resistance of soil $R$ ) by various hyperbolic functions that tend to infinity at a limiting resistance of soil $p_{u}$ (Fig.1).

\footnotetext{
Corresponding author: yarkinvv@mail.ru
} 


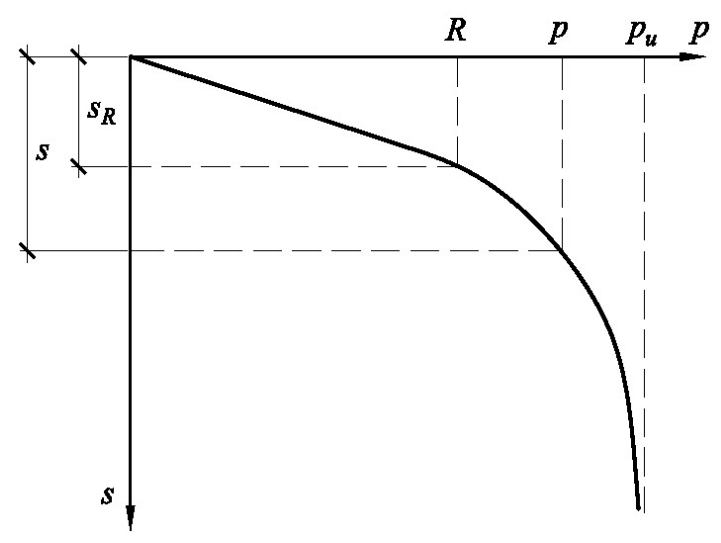

Fig.1. Dependence of settlement on pressure $s=f(p)$

For the first time, a linear-fractional function describing the hyperbolic dependence $s=f(p)$ in the phase of shear was proposed by Popov B.P. based on the well-known Schleicher solution for a linearly elastic half-space [15]. Subsequently, Vyalov S. S. [15] considered exponential and fractional-linear laws of soil deformation, in which a nonlinear settlement is determined either by method of layer-by-layer summation or by the Schleicher equation using the strain modulus $E_{i}=f\left(p_{i}, t\right)$, depending on the load on the base and the time of its action.

Hyperbolic dependence is also used to determine the development of settlement complex organic-rich soils over time [16].

In [17], nonlinear settlement is proposed to be calculated by method of layer-by-layer summation using the nonlinear tangent modulus deformation of soil, determined from the hyperbolic dependence on the stress level.

Using the offer by Popov B.P. Klepikov S.N. [13] proposed the equation:

$$
s=s_{R} \cdot \frac{\left(p_{u}-R\right) \cdot p}{\left(p_{u}-p\right) \cdot R},
$$

where $p$ - average pressure on the foundation base; $R$ - design resistance of soil; $p_{u}-$ limiting resistance (pressure corresponding to the bearing capacity of soil); $s R$ - settlement at pressure $p=R$.

In turn, Malyshev M.V. [4] proposed the equation:

$$
s=s_{R} \cdot\left[1+\frac{\left(p_{u}-R\right) \cdot(p-R)}{\left(p-\sigma_{z g, 0}\right) \cdot\left(p_{u}-p\right)}\right],
$$

where $\sigma_{z g, 0}$ - vertical stress from dead weight pressure of soil in the level of the foundation base.

It should be noted that sometimes in the above equations, instead of $R$ and $S R$, are accepted the initial critical pressure $p_{c r}$ and the corresponding settlement [18].

The main disadvantage of the currently existing methods based on hyperbolic dependence is that, despite confirmation by certain experimental data, they are not universal and have a specific field of application, depending on the kind of soil, its physical and stress-strain state.

Note that for the simplified hyperbolic dependences proposed by Klepikov S.N., Malyshev M.V. and others, a good agreement with the experimental data is observed up to about half of the pressure range $R-p_{u}$, and then the results are significantly overestimated and at $p=p_{u}$ tend to infinity [18]. 


\section{Methods}

This paper proposes a method for determining the nonlinear settlement of the foundation, based on the method of layer-by-layer summation, in which:

- at pressures not exceeding the initial critical pressure $p_{c r}$, the soil is considered as a linearly deformable;

- at pressures in the range from $p_{c r}$ to limiting resistance of the base $p_{u}$ is an elastoplastic body, in which: with increasing base pressure, the dependence of strain on pressure is approximated by a fractional-linear hyperbolic function, and with decreasing pressure - by linear dependence.

The main difference of the proposed method is that the hyperbolic approximation does not apply to the whole massif of soil, as in similar equations Popov B.P., Klepikov S.N., Malyshev M.V. and others, but only to each individual elementary layer (Fig.2). In this case, the total settlement in each elementary layer from pressures exceeding the limiting value for this layer is limited by the layer thickness $h$.

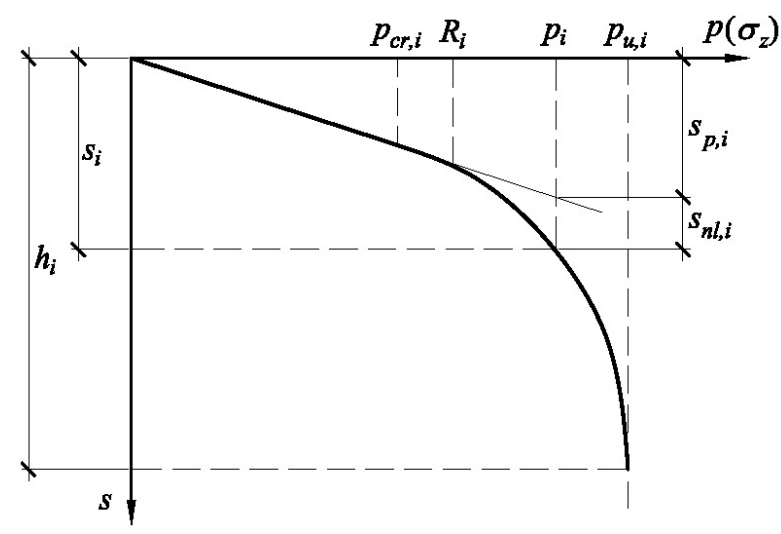

Fig. 2. Calculation scheme for determining the settlement in the elementary layer from the pressure exceeding the initial critical pressure

In accordance with current regulatory documents in determining the vertical component of the ultimate resistance of the base, it is assumed that the base soils below the footing are uniform to a depth not less than the width of the foundation.

Since the increase in settlement in the phase of shear occurs mainly due to lateral displacements of the soil within the depth of development of zones of ultimate equilibrium, in this work, as a simplifying assumption, it is assumed that the first elementary layer has a thickness corresponding to the maximum depth of development of zones of ultimate equilibrium for given parameters of the foundation and properties of soil. In the case of heterogeneous soils within this layer, it is assumed to be conditionally homogeneous with averaged mechanical characteristics.

In accordance with the proposal Yaropolskii I.V. [19] the maximum depth of development of the zones of ultimate equilibrium can be determined by the equation

$$
z_{u}=\frac{b}{2 \cdot \operatorname{tg}(45-\varphi / 2)}
$$

where $b$-width of foundation; $\varphi$ - internal friction angle. 


\subsection{Determination of linear and non-linear components of settlement}

The settlement of foundation with regard only the components depending on the external load on the foundation, is expressed by the equation

$$
s=s_{p}+s_{n l},
$$

where $s_{p}$ - settlement corresponding to the condition of linear deformability of the base; $s_{n l}$ - additional settlement caused by the development of zones of ultimate equilibrium.

Both components are determined by step-by-step interpretation of method of layer-bylayer summation, which leads to the degeneration of elementary layers in the process of solving the problem, which allows us to consider the problem to be solved as geometrically nonlinear.

$$
s_{p}=\sum_{1}^{n} s_{p, i} ; \quad s_{p, i}=\beta \cdot \frac{\sigma_{z p, i}}{E_{i}} \cdot h_{i},
$$

where $n$ - number of elementary layers located within the compressible stratum; $\beta$ - nondimensional coefficient, equal $0.8 ; \sigma_{z p, i}$ - additional vertical stresses at a depth $z_{i}$ from the foundation base; $h_{i}$ and $E_{i}$ - respectively thickness and deformation modulus of the $i$-th elementary layer of soil.

$$
s_{n l}=\sum_{i=1}^{n} s_{n l, i} ; \quad s_{n l, i}=\left(h_{i}-s_{p, i}\right) \cdot k_{i},
$$

where $k_{i}$ - coefficient of approximation to the limit state in the i-th layer, described by a hyperbolic dependence in the form of a linear fractional function with definition domain from 0 to 1 .

The definition domain of the used hyperbolic function $k_{i}$ is assigned from 0 to 1 on the assumption that the total settlement in the i-th elementary layer cannot exceed its thickness and, at stresses in the layer corresponding to the limiting state, is conventionally assumed equal to the layer thickness due to displacing the residual volume of soil to the parties, i.e.:

$$
\begin{array}{ll}
\text { - at } \sigma_{z, i}=\sigma_{z p, i}+\sigma_{z g, i} \leq \sigma_{c r, i} & k_{i}=0 ; \\
\text { - at } \sigma_{z, i}=\sigma_{z p, i}+\sigma_{z g, i} \geq \sigma_{u, i} & k_{i}=1,
\end{array}
$$

where $\sigma_{c r, i}$ and $\sigma_{u, i}-$ stresses corresponding to the initial critical pressure and the limiting resistance of the $i$-th elementary layer, which can be defined as for a conventional foundation with a depth of $d_{z, I}=d+z_{i}$ and width $b_{z, i}$.

$$
b_{z, i}=\sqrt{\frac{p \cdot A}{\sigma_{z p, i}}+a^{2}}-a,
$$

where $A$ - area of foundation base; $a=(l-b) / 2 ; l$ and $b$ - respectively, the length and width of the foundation base.

Limiting the domain of definition of the coefficient $k_{i}$ allows to derive the following equation

$$
k_{i}=\frac{k_{s, i} \cdot \sigma_{u, i}}{\left(1+k_{s, i}\right) \cdot \sigma_{u, i}-\sigma_{z, i}} \cdot \frac{\left(\sigma_{z, i}-\sigma_{c r, i}\right)}{\left(\sigma_{u, i}-\sigma_{c r, i}\right)},
$$

where $k_{s, i}$ - coefficient which depending on the kind and condition of the soil, as well as the parameters of the foundation.

The use of the coefficient $k_{s, i}$ in the equation (8) makes it universal and allows to approximate almost any curve of the dependence of settlement on pressure (Fig. 3). 


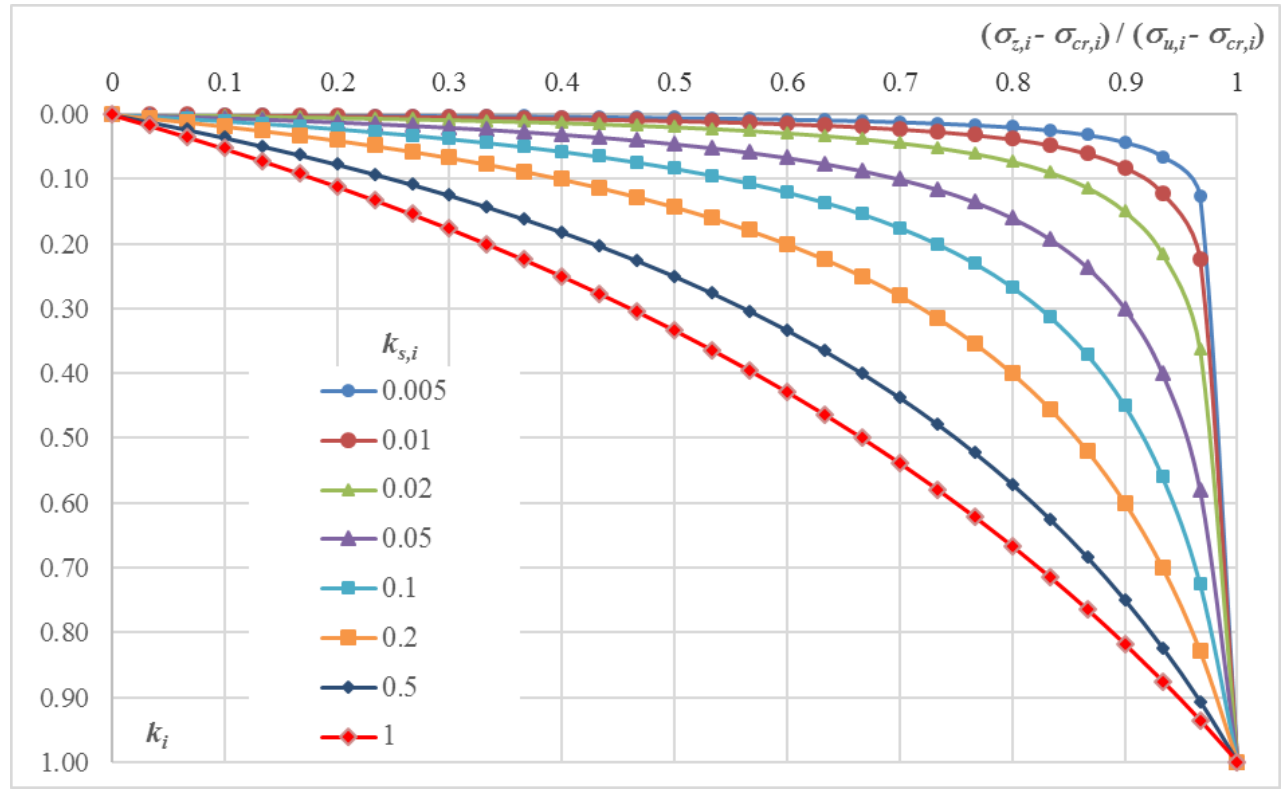

Fig. 3. The dependence of the coefficient $k$ on pressure at various values of $k_{s}$

\subsection{Determination of the coefficient $k_{s}$ influencing the shape of the nonlinear strain curve}

The shape of the curve $s=f(p)$ depends on various factors, among which the most significant are properties of the soil, size, shape and depth of the foundation base. All curves $s=f(p)$ encountered in practice can be divided into three main types [20] (Fig. 4):

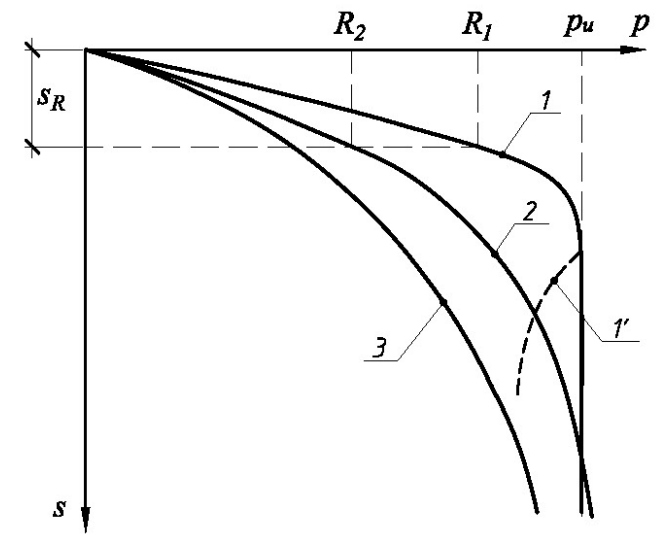

Fig. 4. Characteristic dependences of settlement on pressure for a non-linearly deformable base:

1 - for foundations with a small relative depth and dense sandy soils in the base;

2 - for the bases from sand of average density or clay soil;

3 - for foundations with a large relative depth and bases from loose sand or highly porous watersaturated clay soils

- curves 1 types retain a linear relationship even at pressures close to the limiting one, and after reaching the limiting pressure even unloading does not stop the process of soil extraction. The loss of bearing capacity is always accompanied by raising the surface of the 
soil around the foundation. According to Gorbunov-Posadov M.I., this type of dependence is characteristic of sandy soils [21];

- curves 2 types have a shorter linear section and a longer curved section compared with curves 1 type. Raising the surface of the soil with a loss of bearing capacity may not occur;

- curves 3 types have almost no initial linear segment. Raising the ground surface around the foundation is completely absent.

The magnitude of non-linear deformations decreases with a decrease in the ratio of the sides of the base of the foundations, as well as with an increase in the size and depth of foundations, strength parameters and unit weight of the soil [21].

Analysis of the results of experimental studies performed by the authors on sandy soils [22], as well as the results given in the literature [20, 18], made it possible to recommend the following equation for determining the coefficient $k_{s}$

$$
k_{s, i}=\frac{v_{i}^{2}}{16} \cdot(0.5+0.5 / \eta) \cdot\left(0.5+\frac{\left(d+z_{i}\right)}{b}\right),
$$

where $v$ - Poisson's ratio of soil; $\eta$ - ratio of the sides of the base of the foundation $\eta=l / b$; $d$ - depth of the foundation; $z_{i}$ - depth of the considered point from the bottom of the foundation.

Instead of the ratio of the sides of the base of the foundation in the equation (9), can use the ratio of the perimeter to the area of the base of the foundation with the corresponding coefficient.

It is possible to use formula (9) with other values of the coefficients for the main influencing factors, which are experimentally verified.

\subsection{Correction of additional stresses in elementary layers caused by the development of zones of ultimate equilibrium}

In [23], it was shown that the stress distribution in the soil massif, obtained in accordance with the Boussinesq solution for an elastic half space, can be used in determining nonlinear settlement without a significant loss of accuracy.

Nevertheless, taking into account that settlement caused by shear deformations can reach significant values in addition to physical nonlinearity, it is also necessary to take into account geometric nonlinearity.

Based on the previously made assumption that the settlement $s_{n l, i}$ occurs due to the displacement of the soil to the sides from under the foundation, thereby reducing the thickness $i$-th elementary layer of soil and, accordingly, its distribution capacity, so the depth $z_{i+1}$ for the next elementary layer is adjusted

$$
z_{i+1}=z_{i}+h_{i}-s_{n l, i} \text {. }
$$

Application of the equation (10) leads to a reduction of the intensity fading additional stresses $\sigma_{z p}$ (Fig. 5). 


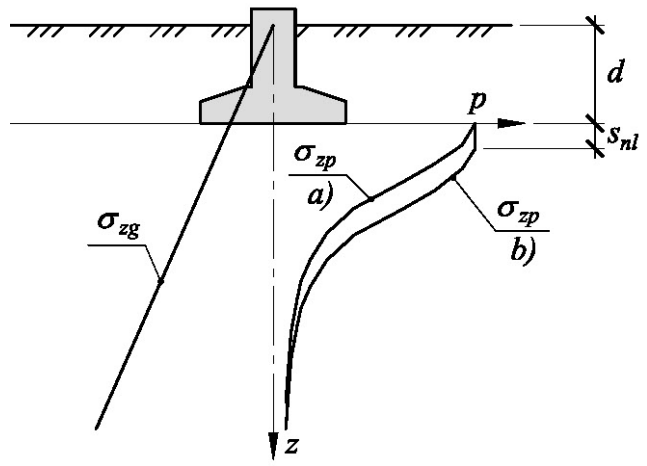

Fig. 5. Stress diagrams in a soil massif at pressures exceeding $R$ : a - stresses which defined without regard to the equation (10); $b$ - stresses which defined with regard to the equation (10)

From Fig. 5 shows that an increase in additional vertical stresses in the ground at $p_{c r}<p$ $<p_{u}$, calculated with regard to equation (10), occurs mainly directly below the foundation in the zone formation of compacted core, and at depth corresponding boundary of compressible stratum is practically absent.

\section{Results and Discussion}

The proposed method was used in analyzing the results of experimental studies of the carrying capacity and deformability of sandy soil in the base of a rigid stamp with dimensions of $1 \times 0.16 \mathrm{~m}$. Design parameters of sandy soil: $\gamma_{\text {sat }}=19.9 \mathrm{\kappa N} / \mathrm{m}^{3} ; \gamma_{\text {unsat }}=16.18$ $\mathrm{\kappa N} / \mathrm{m}^{3} ; v=0.3 ; C=15 \mathrm{kPa} ; \varphi=33^{\circ} ; E=42 \mathrm{MPa}$.

For these conditions, the load on the stamp corresponding to the initial critical pressure was $33.7 \mathrm{kN}$, and the load corresponding to the limiting resistance was $97.8 \mathrm{kN}$.

Also for comparison, calculations were performed using the methods proposed by Malyshev M.V. and Klepikov S.N., and by the finite element method using the elastoplastic model Mohr-Coulomb. The results of the calculations are presented in graphical form in Fig. 6.

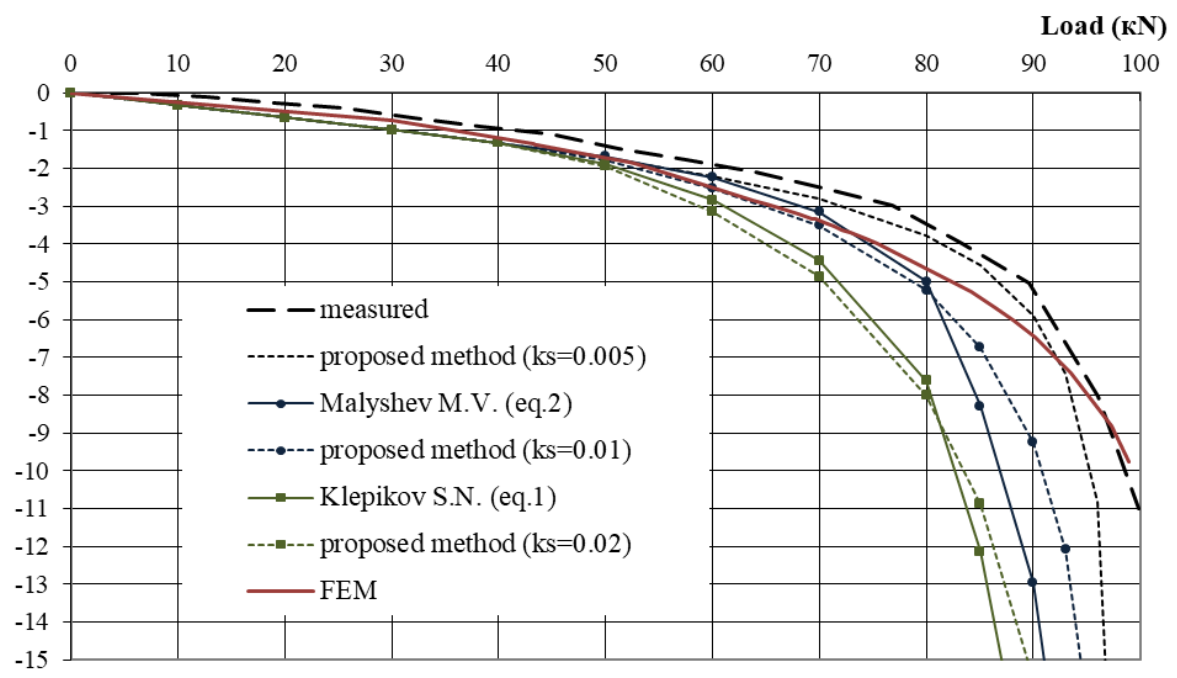

Settlement (mm)

Fig. 6. Comparison of the predicted and measured settlements 


\section{Conclusions}

Analysis of settlement graphs, calculated theoretically and measured experimentally, allows us to draw the following conclusions:

- methods proposed by Malyshev M.V. and Klepikov S.N., provide a significant margin in determining the settlement, and at loads close to the limit value, the settlements determined by these methods reach unrealistic values;

- the best convergence of the results with experimental data was shown by the finite element method and the proposed method with coefficient $k_{s}$ determined by the eq. (9);

- the proposed method allows to determine the elastic-plastic settlements of the foundation over the entire pressure range up to the exhaustion of the bearing capacity of the base taking into account the heterogeneity of the geological structure of the soil massif, including the presence of weak underlying layers of soil;

- the proposed method with $k_{s}=0.01$ and with $k_{s}=0.02$ shows results close to the methods proposed by Malyshev M.V. and Klepikov S.N. respectively.

\section{References}

1. Y. Zaretskii, Soil Mech Found Eng, 19 (1982) https://doi.org/10.1007/BF02336188

2. A. Bugrov, Soil Mech Found Eng, 11 (1974) https://doi.org/10.1007/BF01703812

3. D. Shapiro, Soil Mech Found Eng, 22 (1985) https://doi.org/10.1007/BF01711561

4. M. Malyshev. Soil Mech Found Eng, 33 (1996) https://doi.org/10.1007/BF02354277

5. M. Gol'dshtein, S. Kushner. Soil Mech Found Eng, 7 (1970) https://doi.org/10.1007/BF01704110

6. S. Kushner. Soil Mech Found Eng, 31 (1994) https://doi.org/10.1007/BF02336719

7. S. Klepikov, Y. Slobodyan. Soil Mech Found Eng, 26 (1989) https://doi.org/10.1007/BF02306661

8. A. Pilyagin, S. Kazantsev. Soil Mech Found Eng, 26 (1989) https://doi.org/10.1007/BF02306662

9. A. Fadeev, A. Preger, Soil Mech Found Eng, 21 (1984) https://doi.org/10.1007/BF01710611

10. A. Tregub, Yu. Kirichek. Building constructions, 83(2) (2016)

11.I. Lukić Kristić, V.Szavits-Nossan, P. Miščević. GRAĐEVINAR, 69 (2017) doi: https://doi.org/10.14256/JCE.1926.2016

12. A. Ibrahim, A. Dif, W. Othman, Alexandria Engineering Journal, 57 (2018) https://doi.org/10.1016/j.aej.2016.11.021

13.S.N. Klepikov, Calculation of structures on a deformable base. (NIISK, 1996)

14. D. Rachkov, Ya. Pronozin, V. Chikishev, Magazine of Civil Engineering, 4(72), (2017) doi: 10.18720/MCE.72.5

15. S. Vyalov. Rheological basis of soil mechanics (Higher School, 1978)

16. Mosleh A. Al-Shamrani, Engineering Geology, 77 (2005) doi:10.1016/j.enggeo.2004.07.004

17. G. Yang, Y. Luo, Y. Zhang and E. Wang, Proceedings of the 18th International Conference on Soil Mechanics and Geotechnical Engineering. (2013)

18. V. Lushnikov, A. Yardyakov, Bulletin of the PNRPU Construction and Architecture, 2 (2014) 
19. N. Ryazanov, Soil Mech Found Eng, 6 (1969). https://doi.org/10.1007/BF01711030

20.S. Kushner. Calculation of deformations of the foundations of buildings and structures (IPO Zaporozhye LLC, 2008)

21. A. Pilyagin. Designing the foundations and foundations of buildings and structures (Publishing House ASV, 2006)

22. V. Yarkin, Building constructions, 58 (2003)

23. B. Lehane, M. Fahey, A simplified nonlinear settlement prediction model for foundations on sand. Can. Geotech. J. 39 (2002) https://doi.org/10.1139/t01-091 